
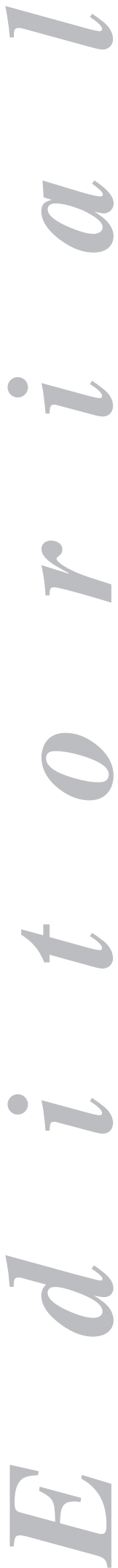

\section{Treating Helicobacter pylori infection - small remedies for great evils?}

Helicobacter pylori infection currently remains a most prevalent infection worldwide. This fact, together with its proven implication in relevant gastrointestinal conditions (peptic ulcer disease, chronic gastritis, gastric adenocarcinoma, and MALT gastric lymphoma) and the fact that we still lack effective vaccination, makes effective therapy availability essential for infected patients eligible for eradication.

Ever since the discovery of $H$. pylori during the late $20^{\text {th }}$ century a number of therapies have been attempted against this infection with various combined antimicrobial drug regimens usually associated with a powerful acid secretion inhibitor, i.e., a proton pump inhibitor (PPI). However, after over 20 years' experience in the management of $H$. pylori infection an ideal therapy remains to be devised. Various expert and consensus meetings have established and supported a number of regimens and therapy sequences for which eradication rates above $80 \%$ with an initial course in "intent-to-treat" protocols were reported $(1,2)$. Most of these guidelines recommend the so-called classic triple therapy including a PPI plus clarithromycin and amoxicillin as first-line therapy. Various meta-analyses and clinical studies including long series of patients have reported that this regimen fails in over $20-30 \%$ of patients, and more importantly that eradication failure rates seem to be on the rise (3). The main reason for this loss of effectiveness when using the classic triple therapy is bacterial resistance to clarithromycin, which is increasing significantly in several geographical areas, and already approaches $20 \%$ in our setting (4).

Different treatment options have been suggested during the last few years in an attempt to increase the effectiveness of eradicating therapy against $H$. pylori infection. A regimen including a new fluorquinolone, levofloxacin, plus amoxicillin and a PPI is particularly promising. Levofloxacin, a levo isomere of ofloxacin, is an antibacterial agent with wide-spectrum activity against Gram-positive and Gram-negative organisms, including high in vitro effectiveness against $H$. pylori. Its rapid absorption following oral administration is scarcely disturbed by concomitant food ingestion, and its high bioavailability approaches $100 \%$ (5). Most studies evaluating the eradicating effectiveness of $H$. pylori infection with levofloxacin-based regimens have been performed in patients failing to respond to prior therapy; these regimens were used as second-line rescue therapies, and demonstrated higher eradicating effectiveness and improved tolerability versus quadruple therapy (PPI, metronidazole, bismuth, tetracycline), a second-line regimen usually recommended by guidelines $(1,2)$. However, studies evaluating combined levofloxacin, clarithromycin, and a PPI as first-line therapy to eradicate $H$. pylori infection are very few, and most include a small number of patients. 
With all the above, Castro-Fernández et al. (6) report in this issue a prospective, observational, single-center study evaluating the eradicating effectiveness of a firstline triple regimen with a PPI, levofloxacin, and clarithromycine against $H$. pylori infection. They include successive patients with dyspeptic symptoms and H.pylori infection (as identified using the $\mathrm{C}^{13}$-urea breath test, rapid urease test, or histological examination). Patients younger than 18 years with severe comorbidities, history of prior eradicating therapy, gastric surgery, or allergy to study drugs were excluded. Patients included received omeprazole $20 \mathrm{mg} / 12 \mathrm{~h}$ or another PPI at equivalent doses, levofloxacin $500 \mathrm{mg} / 12 \mathrm{~h}$, and amoxicillin $500 \mathrm{mg} / 12 \mathrm{~h}$ for 10 days. Compliance was assessed using specific interviewing, and eradication was considered successful in the presence of a negative $\mathrm{C}^{13}$-urea breath test at 6-8 weeks after treatment completion.

The study included 135 patients (mean age: 53 years, $65 \%$ female) $-48.9 \%$ with non-investigated dyspepsia, 36.3 with functional dyspepsia, and 14.8 with peptic ulcer disease; 130 patients completed the study's therapy and protocol. Five patients $(3.7 \%)$ discontinued therapy because of adverse events. Adverse events occurred in 16 patients (11.8\%), and all were mild (nausea was most common) except for one case (seizures). In the per-protocol analysis efficacy was achieved for 97 of 130 patients (74.6\%; $95 \%$ CI: $67.1-82.1 \%$ ), and in 97 of 135 patients in the intention-totreat population (71.8\%; 95\% CI: $64.2-79.4 \%$ ). Eradication rates were similar for patients with non-investigated dyspepsia, functional dyspepsia, or peptic ulcer disease.

As previously mentioned, few studies have been reported thus far where efficacy was analyzed for a regimen including a PPI, levofloxacin, and amoxicillin as firstline therapy against $H$. pylori infection. Only six such studies have been reported in the literature to this day (7-12). Eradication rates in all these studies were very high, above $80 \%$ : $83(12), 87(7), 90(9), 91(11)$, and $92 \%(8,10)$. Only one of these studies was carried out in our country (12).

The study by Castro-Fernández et al. is the second such study in Spain, and the one with a higher number of patients. The eradication rate obtained against $H$. pylori infection (72\%) is strikingly lower than described in the above-mentioned trials, inferior to $80 \%$ in the intention-to-treat population, which is considered the minimum percentage acceptable for a regimen according to consensus meetings $(1,2)$. The other study performed in our country by Gisbert et al. also reported an eradication rate lower than those obtained in other publications (83\%), but above the minimum acceptable eradication threshold of $80 \%$. Data reported by both studies probably result from increased bacterial resistance to levofloxacin in our setting. Bacterial resistance in countries with high quinolone use is known to be high and still on the rise. Furthermore, eradication rates with this drug have been shown to substantially decrease when bacterial resistance is acknowledged. Thus, the use of levofloxacin in first-line eradicating regimens is only recommended in areas where primary resistance is known to be low, or after antibiotic susceptibility studies to rule out resistance (13).

In our country, these data should prompt consideration of the real convenience of using levofloxacin-based triple therapy as first-line regimen against $H$. pylori infection rather than the regimen laid down by consensus guidelines, which includes clarithromycin, amoxicillin, and a PPI $(1,2)$. While in vitro $H$. pylori resistance to quinolones as currently reported in Spain is $6 \%$, much lower than to clarithromycin (43\%) and metronidazole (42\%) (14), clinical studies available thus far reveal that first-line levofloxacin-based triple therapy is not much more beneficial than the 
classic regimen using clarithromycin. As appropriately pointed out by Castro-Fernández et al. during their discussion of the results obtained, attempting other therapy options is likely advisable before initiating this treatment as an alternative to classic triple therapy with clarithromycin as first-line eradication regimen.

In this regard various approaches have been described in recent years that led, significantly at times, to increased eradication rates with the classic triple therapy. One option is using a PPI dose twice as high as usual in triple therapy when duration is seven days; this significantly increases eradication rates (82 vs. 74\%, 95\% CI: 1.01-1.17) (15). Another potentially effective strategy is prolonging the classic triple therapy's duration from seven to ten days, particularly in patients with peptic non-ulcer disease; eradication rates also increase significantly in non-ulcer patients (66 vs. $77 \%, p=0.08)(16)$. Administering a probiotic added to classic triple therapy effectively reduces diarrhea as commonly associated with this therapy, and increases eradication rates (83.6 vs. 74.8\%; OR: 1.84, 95\% CI: 1.34-2.54) (17). Socalled sequential therapy (a PPI and amoxicillin for 5 days followed by a PPI associated with clarithromycin and metronidazole for 5 days), already described by Zullo et al. in 2000 (18), is recently considered a good first-line alternative to triple therapy in some studies, whether the latter is administered for 7 or 10 days (19).

Last, but not least, the effectiveness of any therapy prescribed for a disease greatly depends on good patient compliance - not only regarding the administration of prescribed doses, but also dosage intervals and overall therapy duration. In our present case, the complexity of some eradicating therapies against $H$. pylori infection, and their commonly associated, usually mild side effects, makes it necessary for prescribing physicians to let patients know that treatment effectiveness is greatly influenced by patient adherence. In this respect devoting a few minutes to carefully discuss treatment with patients in a way that they can understand, and to make sure that they did understand it, is important as these "spent" time will undoubtedly be of great advantage for $H$. pylori eradication in the long run, particularly in a time when increased bacterial resistance may significantly complicate the resolution not only of diseases associated with this bacterium but also of most infections.

\author{
C. Martín de Argila de Prados \\ Department of Gastroenterology. Hospital Universitario Ramón y Cajal. \\ Madrid, Spain
}

\title{
REFERENCES
}

1. Malfertheiner P, Megraud F, O’Morain C, Bazzoli F, El-Omar E, Graham D, et al. Current concepts in the management of Helicobacter pylori infection: the Maastricht III Consensus Report. Gut 2007; 56: 772-81.

2. Monés J, Gisbert JP, Borda F, Domínguez-Muñoz E; Grupo Conferencia Española de Consenso sobre Helicobacter pylori. Indications, diagnostic tests and Helicobacter pylori eradication therapy. Recommendations by the $2^{\text {nd }}$ Spanish Consensus Conference. Rev Esp Enferm Dig 2005; 97: 348-74.

3. Vakil N, Mégraud F. Eradication therapy for Helicobacter pylori. Gastroenterology 2007; 133: 985-1001.

4. Mégraud F. H pylori antibiotic resistance: prevalence, importance, and advances in testing. Gut 2004; 53: 1374-84.

5. Anderson VR, Perry CM. Levofloxacin: a review of its use as a high-dose, short-course treatment for bacterial infection. Drugs 2008; 68: 535-65.

6. Castro-Fernández M, Lamas E, Pérez-Pastor A, Pabón M, Aparcero R, Vargas-Romero J, et al. Efficacy of triple therapy with a proton pump inhibitor, levofloxacin, and amoxicillin as first-line treatment to eradicate Helicobacter pylori. Rev Esp Enferm Dig 2009; 101(6): 395-402. 
7. Antos D, Schneider-Brachert W, Bästlein E, Hänel C, Haferland C, Buchner M, et al. 7-day triple therapy of Helicobacter pylori infection with levofloxacin, amoxicillin, and high-dose esomeprazole in patients with known antimicrobial sensitivity. Helicobacter 2006; 11: 39-45.

8. Cammarota G, Cianci R, Cannizzaro O, et al. Efficacy of two one-week rabeprazole/levofloxacin-based triple therapies for Helicobacter pylori infection. Aliment Pharmacol Ther 2000; 14: 1339-43.

9. Di Caro S, Zocco MA, Cremonini F, Candelli M, Nista EC, Bartolozzi F, et al. Levofloxacin based regimens for the eradication of Helicobacter pylori. Eur J Gastroenterol Hepatol 2002; 14: 1309-12.

10. Marzio L, Coraggio D, Capodicasa S, Grossi L, Cappello G. Role of the preliminary susceptibility testing for initial and after failed therapy of Helicobacter pylori infection with levofloxacin, amoxicillin, and esomeprazole. Helicobacter 2006; 11: 237-42.

11. Rispo A, Di Girolamo E, Cozzolino A, Bozzi R, Morante A, Pasquale L. Levofloxacin in first-line treatment of Helicobacter pylori infection. Helicobacter 2007; 12: 364-5.

12. Gisbert JP, Bermejo MF, Infante JM, Gallardo BP, Bermejo AB, Rodríguez JM, et al. Levofloxacin, amoxicillin, and omeprazole as first-line triple therapy for helicobacter pylori eradication. J Clin Gastroenterol 2009; 43: 384-5.

13. Cattoir V, Nectoux J, Lascols C, Deforges L, Delchier JC, Megraud F, et al. Update on fluoroquinolone resistance in Helicobacter pylori: new mutations leading to resistance and first description of a gyrA polymorphism associated with hypersusceptibility. Int J Antimicrob Agents 2007; 29: 389-96.

14. Cibrelus LA, Pérez de Ayala A, Alarcón T, Urruzuno P, Martínez MJ, López-Brea M. In vitro efficiency of ciprofloxacin and rifampicin as potential second-line treatment in Spanish Helicobacter pylori clinical isolates. Helicobacter 2006; 11: 402-3.

15. Villoria A, García P, Calvet X, Gisbert JP, Vergara M. Meta-analysis: high-dose proton pump inhibitors vs. standard dose in triple therapy for Helicobacter pylori eradication. Aliment Pharmacol Ther 2008; 28: 86877.

16. Calvet X, Ducons J, Bujanda L, Bory F, Montserrat A, Gisbert JP; Hp Study Group of the Asociación Española de Gastroenterología. Seven versus ten days of rabeprazole triple therapy for Helicobacter pylori eradication: a multicenter randomized trial. Am J Gastroenterol 2005; 100: 1696-701.

17. Tong JL, Ran ZH, Shen J, Zhang CX, Xiao SD. Meta-analysis: the effect of supplementation with probiotics on eradication rates and adverse events during Helicobacter pylori eradication therapy. Aliment Pharmacol Ther 2007; 25: 155-68.

18. Zullo A, Rinaldi V, Winn S, Meddi P, Lionetti R, Hassan C, et al. A new highly effective short-term therapy schedule for Helicobacter pylori eradication. Aliment Pharmacol Ther 2000; 14: 715-8.

19. Moayyedi P. Sequential regimens for Helicobacter pylori eradication. Lancet 2007; 370: 1010-2. 